\title{
A Geometric Characterisation of Resonance in Hopf Bifurcation from Relative Equilibria
}

\author{
David Chan Ian Melbourne \\ Department of Mathematics and Statistics \\ University of Surrey \\ Guildford \\ Surrey, GU2 7XH \\ UK \\ 30 June, 2007

\begin{abstract}
We give a new characterisation of resonance in Hopf bifurcation from relative equilibria in systems with compact symmetry group. This characterisation provides a full geometric explanation of the resonance phenomenon. In addition, we develop techniques based on normal form theory to give a complete solution to the associated bifurcation problem.
\end{abstract}

\section{Introduction}

Spiral waves in planar excitable media exhibit intriguing dynamics, some of which is now well-understood from the point of view of dynamical systems with symmetry. The underlying symmetry group is $\mathbf{S E}(2)$ (rotations and translations in the plane) and the simplest possible solution is a relative equilibrium where the time evolution is a rigid rotation in the plane.

Many people observed the onset of quasiperiodic behaviour where the tip of the spiral wave traces out a two-frequency "flower" pattern instead of a perfect circle. This behaviour was termed meander by Winfree [18]. Barkley et al. [3] and Karma [12] pointed out Hopf bifurcation as a mechanism for meander, and this was confirmed experimentally for the Belousov-Zhabotinskii reaction by Li et al. [14]. The analysis to this point makes use only of the group $\mathbf{S O}(2)$ of rotations (though we note that the distinctive nature of the flower patterns associated with meander is due to the full $\mathbf{S E}(2)$ symmetry [11]) and is in accordance with the theory of Krupa [13] for 
local bifurcations from relative equilibria in systems with compact symmetry. The bifurcating "modulated rotating waves" are examples of relative periodic solutions.

Numerical experiments [1] and chemical experiments [14] exhibited a codimension two point where the Hopf frequency equals the frequency of the rotating spiral wave and the meandering motion of the spiral tip is replaced by linear drift. In a landmark paper, Barkley [2] correctly explained this phenomenon as a new type of resonance in systems with Euclidean symmetry.

The first mathematically rigorous results on the transition to linearly drifting spirals were obtained by Wulff [19], and these results were further developed in [8, 10, 11, 16]. In particular, Fiedler et al. [8] generalised the theory of Krupa [13] to apply to general finite-dimensional Lie groups, provided the isotropy subgroup of the underlying relative equilibrium is compact. (This condition is certainly satisfied for spiral waves.)

Subsequently, Wulff [20] extended the rigorous analysis in [19] to general finitedimensional Lie groups, including the group $\mathbf{S O}(3)$. This example is relevant to the behaviour of spiral waves on a sphere and the resonance phenomenon is very similar to the planar case. The underlying spiral wave rotates rigidly on the surface of the sphere about an axis through the centre of the sphere, and Hopf bifurcation leads to two-frequency meandering about a typically nearby axis. At a resonance (where the Hopf frequency equals the rotation frequency) the meander is about an axis almost perpendicular to the original axis. See also $[6,7]$.

From the point of view of doing calculations, the case of compact $\mathbf{S O}(3)$ symmetry is much more difficult than the noncompact $\mathbf{S E}(2)$ case - $\mathbf{S E}(2)$ is solvable and the equations of motion along the group orbit can hence be solved explicitly by successive integrations and Fourier analysis [10]; in contrast $\mathbf{S O}(3)$ is semisimple. Chan [6] used the normal form technique of Fiedler \& Turaev [9] to overcome the problem of semisimplicity and obtained an elegant solution to the $\mathbf{S O}(3)$ case.

There still remained a lack of clarity of the nature of the resonance phenomenon itself. Fiedler \& Turaev [9] gave a Lie algebraic definition of resonance in terms of the structure of equations when transformed to normal form: degeneracies leading to the existence of extra monomials in the normal form equations were termed resonances. Wulff [20, Definition 2.5] gave a more geometric but still infinitesimal Lie-theoretic definition of resonance which is equivalent to the condition in [9]. However, neither paper establishes a link between the definition and the observed dynamical phenomena.

In this paper, we focus on the situation where the group of symmetries is a compact Lie group. We give a geometric definition of resonance, based on the theory of maximal tori, that agrees perfectly (and provably) with the observed global dynamics. We note that in both the $\mathbf{S E}(2)$ and $\mathbf{S O}(3)$ examples, typically the bifurcating relative periodic solution is close (as a set in phase space) to the underlying relative equilibrium. It is precisely at a resonance that this closeness breaks down. In Theorem 3.8, we prove that our definition of resonance is equivalent to this setwise continuity prop- 
erty for the dynamics. In Proposition 3.10, we prove that our definition coincides with the definitions in $[9,20]$. A consequence is that resonance is a codimension two phenomenon for every nonabelian compact Lie group (and is impossible for abelian groups).

In the remainder of the introduction, we sketch our approach to resonance. More background and full details can be found in the ensuing sections. Recall that a relative equilibrium is a solution of the form $x(t)=\exp (t \eta) x(0)$ where $\eta$ is a Lie algebra element. A relative periodic solution is a solution $y(t)$ that is not a relative equilibrium and satisfies $y(T)=\sigma y(0)$ for some $T>0$. The least such $T$ is called the (relative) period and $\sigma$ is called a spatiotemporal generator.

Hopf bifurcation from a relative equilibrium $x(t)=\exp (t \eta) x(0)$ leads to a branch of relative periodic solutions $y_{\lambda}(t)$ satisfying $y_{\lambda}\left(T_{\lambda}\right)=\sigma_{\lambda} y_{\lambda}(0)$, where $T_{\lambda} \rightarrow 2 \pi / \omega_{b}$ as $\lambda \rightarrow 0$ and $\omega_{b}>0$ is the Hopf frequency. The relationship between $\eta$ and $\sigma_{\lambda}$ (see Proposition 2.1) is that

$$
\sigma_{\lambda} \rightarrow \sigma_{0}=\exp \frac{2 \pi}{\omega_{b}} \eta
$$

Typically, $\sigma_{0}$ is a regular element lying in a unique maximal torus. In this case, we say that the Hopf bifurcation is nonresonant. Otherwise, $\sigma_{0}$ is singular and we say that the Hopf bifurcation is resonant.

In other words, resonance occurs precisely when the Hopf frequency $\omega_{b}$ is such that $\exp \frac{2 \pi}{\omega_{b}} \eta$ is a singular element of $G$.

Our main aim in this paper is to clarify the phenomenon of resonance by introducing the above definition and connecting it to the dynamics. A secondary purpose is to use the equivalence with the definition of Fiedler \& Turaev to tie this in with Chan's use of normal form theory. In particular, we extend Chan's approach in the $\mathbf{S O}(3)$ case to give a complete treatment of Hopf bifurcation (nonresonant and resonant) from relative equilibria with trivial isotropy subgroup in systems with compact symmetry.

Remark 1.1 (a) In principle, the results in this paper could be extended to systems with general noncompact symmetry group, based on a study of the dependence of (the closure of) a topologically cyclic subgroup on its generator. Additional technical issues to be overcome are the possible noncompactness of such subgroups, and the fact that the exponential map need not be onto. For certain groups such as the universal cover of $\mathbf{S L}(2, \mathbb{R})$, there are elements for which no power lies in the image of the exponential map [15, p. 164], so that Floquet-theoretic arguments are problematic. In the absence of motivating examples, we have chosen to restrict to the case of compact symmetry groups where the theory is particularly clean and illuminating.

(b) An important class of examples for applications is Euclidean-type groups of the form $G \ltimes \mathbb{R}^{n}$ where $G$ is a compact Lie group acting on $\mathbb{R}^{n}$. It is possible to make progress on an ad-hoc basis given that the issues for $G$ are already solved in this paper and there are no theoretical difficulties arising from the $\mathbb{R}^{n}$ factor. Originally, we 
intended to cover Euclidean-type groups here, but were aware of the danger of an adhoc approach without sufficient fully worked-out examples; the absence of theoretical difficulties does not exclude interesting phenomena. This was confirmed by recent somewhat surprising calculations of Chan [5] in the case of the Euclidean group $\mathbf{S E}(3)$ (isometries of three-dimensional space). We hope to explore the ramifications of these calculations more carefully in future work.

The remainder of the paper is organised as follows. Section 2 contains background material on Hopf bifurcation from relative equilibria. In Section 3, we give our geometric characterisation of resonance. In Section 4, we use normal form theory to solve the bifurcation equations at a nonresonant or resonant Hopf bifurcation.

\section{Hopf bifurcation from relative equilibria}

Let $G$ be a Lie group and let $X$ be a finite-dimensional vector space. Consider parametrised skew product equations on $G \times X$ of the form

$$
\dot{g}=g \xi(x, \lambda), \quad \dot{x}=f(x, \lambda)
$$

where $\xi: X \times \mathbb{R} \rightarrow L G, f: X \times \mathbb{R} \rightarrow X$ are smooth maps, and $\lambda \in \mathbb{R}$ is a bifurcation parameter. Denote solutions of these equations by $\left(g_{\lambda}(t), x_{\lambda}(t)\right)$. We assume that $g_{\lambda}(0)=e$. (The initial condition $x_{\lambda}(0)$ is of little consequence here.)

Set $\xi(0,0) \equiv \eta, f(0,0) \equiv 0$, so that at $\lambda=0$ there is the relative equilibrium

$$
\left(g_{0}(t), x_{0}(t)\right)=(\exp t \eta, 0) .
$$

Suppose that the relative equilibrium undergoes a Hopf bifurcation at $\lambda=0$. Generically, after centre manifold reduction, we may suppose that $X=\mathbb{R}^{2} \cong \mathbb{C}, f(0, \lambda) \equiv 0$, and $(d f)_{0, \lambda}=\alpha(\lambda)+i \beta(\lambda)$, where $\alpha(0)=0, \alpha^{\prime}(0)>0, \beta(0)=\omega_{b}>0$. We call $\omega_{b}$ the Hopf frequency.

The Hopf theorem guarantees that generically there exists a unique branch of periodic solutions $x_{\lambda}(t)$ for the $\dot{x}$-equation with period $2 \pi / \omega_{b}(\lambda)$ where $\omega_{b}(0)=\omega_{b}$. To fix notation, we suppose that the branch of periodic solutions is supercritical $(\lambda>0)$.

The periodic solution $x_{\lambda}(t)$ corresponds to a relative periodic solution $\left(g_{\lambda}(t), x_{\lambda}(t)\right)$ for equation (2.1) with relative period $2 \pi / \omega_{b}(\lambda)$ and spatiotemporal generator $\sigma_{\lambda}=$ $g_{\lambda}\left(\frac{2 \pi}{\omega_{b}(\lambda)}\right)$.

Proposition $2.1 \lim _{\lambda \rightarrow 0^{+}} \sigma_{\lambda}=\sigma_{0}$ where $\sigma_{0}=\exp \frac{2 \pi}{\omega_{b}} \eta$.

Proof Write $T_{\lambda}=2 \pi / \omega_{b}(\lambda)$ and $T_{0}=2 \pi / \omega_{b}$. It follows from a standard mean value theorem argument that $g_{\mu}\left(T_{\lambda}\right) \rightarrow g_{\mu}\left(T_{0}\right)$ uniformly in $\mu \in\left[0, \mu_{0}\right]$ for $\mu_{0}>0$ 
sufficiently small. Therefore

$$
\begin{aligned}
\left|\sigma_{\lambda}-\sigma_{0}\right| & \leq\left|g_{\lambda}\left(T_{\lambda}\right)-g_{\lambda}\left(T_{0}\right)\right|+\left|g_{\lambda}\left(T_{0}\right)-g_{0}\left(T_{0}\right)\right| \\
& \leq \sup _{\mu \in\left[0, \mu_{0}\right]}\left|g_{\mu}\left(T_{\lambda}\right)-g_{\mu}\left(T_{0}\right)\right|+\left|g_{\lambda}\left(T_{0}\right)-g_{0}\left(T_{0}\right)\right| \rightarrow 0,
\end{aligned}
$$

as $\lambda \rightarrow 0^{+}$.

Note that $\eta \in L G$ determines the long-term behaviour of the relative equilibrium $\left(g_{0}(t), x_{0}(t)\right)$ whereas $\sigma_{\lambda} \in G$ determines the long-term behaviour of the relative periodic solutions $\left(g_{\lambda}(t), x_{\lambda}(t)\right)$. Proposition 2.1 describes the connection between $\sigma_{\lambda}$ and $\eta$.

Convergence in phase space In the Hopf bifurcation in the $\dot{x}$-equation, the branch of periodic solutions consists of loops $S_{\lambda}=\left\{x_{\lambda}(t): t \in \mathbb{R}\right\} \subset X$ that satisfy $S_{\lambda} \rightarrow 0$ (uniformly) as $\lambda \rightarrow 0^{+}$. Similarly, the relative equilibria and relative periodic solutions define flow-invariant sets

$$
E=\overline{\left\{\left(g_{0}(t), 0\right): t \in \mathbb{R}\right\}} \subset G \times\{0\}, \quad P_{\lambda}=\overline{\left\{\left(g_{\lambda}(t), x_{\lambda}(t)\right): t \in \mathbb{R}\right\}} \subset G \times S_{\lambda},
$$

in the phase space $G \times X$. However, $P_{\lambda}$ need not converge to $E$ in any reasonable sense as $\lambda \rightarrow 0^{+}$. We will argue that this nonconvergence characterises resonance.

Remark 2.2 For compact Lie groups, the exponential map exp : $L G \rightarrow G$ is surjective. Hence we have the Floquet representation

$$
g_{\lambda}(t)=\exp \left(\eta_{\lambda} t\right) R_{\lambda}(t)
$$

where $\eta_{\lambda} \in L G$ is chosen so that $\sigma_{\lambda}=\exp \eta_{\lambda}$, and $R_{\lambda}: \mathbb{R} \rightarrow G$ is $2 \pi / \omega_{b}(\lambda)$-periodic.

Again, there need not exist choices such that $\eta_{\lambda} \rightarrow \eta$ as $\lambda \rightarrow 0^{+}$.

\section{Resonance in compact Lie groups}

In this section, we specialise the relative Hopf bifurcation in Section 2 to the case when $G$ is a compact connected Lie group. In Subsection 3.1, we recall background material on maximal tori in $G$. In Subsection 3.2, we define resonance and we characterise resonant bifurcations in terms of the nonconvergence of the relative periodic solutions $P_{\lambda}$ to the underlying relative equilibrium $E$.

Throughout, the Lie group $G$ and its Lie algebra $L G$ are identified with sets of $n \times n$ matrices. We have the adjoint actions $\mathrm{Ad}: G \rightarrow \operatorname{Aut}(L G)$ and $\operatorname{ad}: L G \rightarrow \operatorname{End}(L G)$ given by $\operatorname{Ad}_{g}(\xi)=g \xi g^{-1}(g \in G, \xi \in L G)$ and $\operatorname{ad}_{\eta}(\xi)=\eta \xi-\xi \eta(\eta, \xi \in L G)$ respectively. 


\subsection{Background on maximal tori}

Here, we collect some standard results about maximal tori in compact connected Lie groups $[4,17]$.

Let $G$ be a compact connected Lie group with metric||. A torus $\mathbb{T}$ contained in $G$ is maximal if it is not contained in a torus of larger dimension. Every element of $G$ lies in at least one maximal torus, and all maximal tori are conjugate. The common dimension of the maximal tori is called the rank of $G$, denoted $\operatorname{rank} G$.

Associated to each $g \in G$ is the closed subgroup $H(g)=\overline{\left\{g^{n}: n \in \mathbb{Z}\right\}}$. Such subgroups $H$ are called topologically cyclic and have the form $H \cong \mathbb{T}^{p} \times \mathbb{Z}_{q}$ for some $0 \leq p \leq \operatorname{rank} G$ and $q \geq 1$. The set of $g$ for which $H(g)$ is a maximal torus is residual and of full Haar measure in $G$.

Similarly, each Lie algebra element $\xi \in L G$ generates a one-parameter subgroup in $G$, the closure of which is a torus. The torus is maximal for $\xi$ lying in a residual set of full Lebesgue measure in $L G$.

Definition 3.1 An element $g \in G$ is regular if $g$ lies in a unique maximal torus. Otherwise, $g$ is called singular.

Let $U \subset G$ be the set of regular elements. Then $U$ is open and dense in $G$ with full Haar measure, and

$$
\{g \in G: H(g) \text { is a maximal torus }\} \subset U,
$$

Moreover, the set $G-U$ of singular elements has codimension at least three in $G[17$, Theorem VIII.7.7].

Let $Z(g)=\{h \in G: g h=h g\}$ denote the centraliser of $g$. Then $\operatorname{dim} Z(g) \geq$ $\operatorname{rank} G$ with equality if and only if $g$ is regular, in which case $Z(g)^{0}$ is the unique maximal torus $\mathbb{T}_{g}$ containing $g$. Moreover, $Z(g)^{0}$ is a torus if and only if it is a maximal torus. (Here, $Z(g)^{0}$ denotes the connected component of $Z(g)$ containing the identity.)

Example 3.2 If $G$ is abelian, for example $G=\mathbf{S O}(2)$, then $G$ is a torus and hence there is a unique maximal torus in $G$, namely $G$ itself. In particular, every element of $G$ is regular.

The next simplest case is the group $G=\mathbf{S O}(3)$, the group of $3 \times 3$ orthogonal matrices with determinant 1 . Let $g$ be a nonidentity element of $\mathbf{S O}(3)$. By Euler's rotation theorem, $g$ is a rotation about some axis and we can choose coordinates so that $g=\left(\begin{array}{ccc}\cos \theta & -\sin \theta & 0 \\ \sin \theta & \cos \theta & 0 \\ 0 & 0 & 1\end{array}\right)$ for some $\theta \in(0,2 \pi)$. Any torus containing $g$ lies in $Z(g)^{0}$, so let $h \in Z(g)^{0}$. Then $h$ preserves the eigenspaces of $g$ so $h=h_{1} \oplus h_{2}$ where $h_{1}$ is a $2 \times 2$ matrix commuting with $\left(\begin{array}{rr}\cos \theta & -\sin \theta \\ \sin \theta & \cos \theta\end{array}\right)$ and $h_{2}= \pm 1$. Since $h \in Z(g)^{0}$, 
we must have $h_{2}=1$ and then $h_{1}=\left(\begin{array}{rr}\cos \varphi & -\sin \varphi \\ \sin \varphi & \cos \varphi\end{array}\right)$ for some $\varphi \in[0,2 \pi)$. This shows that $Z(g)^{0} \cong \mathbf{S O}(2)$, so $g$ lies in a unique maximal torus and this torus has dimension 1. Hence $\operatorname{rank} \mathbf{S O}(3)=1$ and the only singular element of $\mathbf{S O}(3)$ is the identity element.

For $A, B \subset G$, define the (nonsymmetric) distance from $\mathrm{A}$ to $\mathrm{B}$,

$$
\operatorname{dist}(A, B)=\sup _{a \in A} \inf _{b \in B}|a-b| .
$$

The set $\mathcal{P}^{G}$ of subsets of $G$ is a metric space with Hausdorff metric

$$
d(A, B)=\operatorname{dist}(A, B)+\operatorname{dist}(B, A) .
$$

Proposition 3.3 The map $U \rightarrow \mathcal{P}^{G}$ given by $g \mapsto \mathbb{T}_{g}$ is continuous.

Proof Note that $\mathbb{T}_{g}=Z(g)^{0}=\exp \operatorname{ker}\left(\operatorname{Ad}_{g}-I\right)$. As a vector space, $\operatorname{ker}\left(\operatorname{Ad}_{g}-I\right)$ depends continuously on $g$ except at changes in dimension, but $\operatorname{dim} \operatorname{ker}\left(\operatorname{Ad}_{g}-I\right)$ is constant for $g \in U$. Moreover $\operatorname{ker}\left(\operatorname{Ad}_{g}-I\right)=L \mathbb{T}_{g}$ for $g \in U$. Hence $g \mapsto L \mathbb{T}_{g}$ is continuous (on $U$ ). To make this precise, put the Hausdorff metric on compact subsets of $L G$. Then $g \mapsto L \mathbb{T}_{g} \cap C$ is continuous in this metric for all compact subsets $C \subset L G$.

By the uniform continuity of exp on compact subsets, it follows that $g \mapsto$ $\exp \left(L \mathbb{T}_{g} \cap C\right)$ is continuous. Choose $C \subset L G$ to be an Ad-invariant compact neighbourhood of 0 . Given $S \subset G$ and $n \geq 1$, define $S^{n}=\left\{g^{n}: g \in S\right\}$. For a fixed maximal torus $\mathbb{T}$, there exists $n \geq 1$ such that $(\exp (L \mathbb{T} \cap C))^{n}=\mathbb{T}$. Since $C$ is Ad-invariant and all maximal tori are conjugate, it follows that $n$ does not depend on the choice of maximal torus. Hence $g \mapsto\left(\exp \left(L \mathbb{T}_{g} \cap C\right)\right)^{n}=\mathbb{T}_{g}$ is continuous.

Thus, $\mathbb{T}_{g}$ depends continuously on $g \in U$. Moreover, $G-U$ consists of discontinuity points in the following sense. If $g_{\lambda}^{1}$ and $g_{\lambda}^{2}$ are smooth curves in $G$ with $g_{0}^{1}=g_{0}^{2}$ singular, then typically: (i) $g_{\lambda}^{1}, g_{\lambda}^{2} \in U$ for $\lambda \neq 0$ small, and (ii) both $\operatorname{dist}\left(T_{g_{\lambda}^{1}}, T_{g_{\lambda}^{2}}\right) \nrightarrow 0$ and $\operatorname{dist}\left(T_{g_{\lambda}^{2}}, T_{g_{\lambda}^{1}}\right) \nrightarrow 0$ as $\lambda \rightarrow 0$.

\subsection{Definition of resonance}

We return to the skew-product system (2.1). Associated to the relative equilibrium solution $(\exp t \eta, 0)$ in $(2.2)$ is the torus $\mathbb{T}_{0}=\overline{\{\exp t \eta: t \in \mathbb{R}\}} \subset G$. We assume throughout that $\mathbb{T}_{0}$ is a maximal torus. In particular

$$
\text { ker } \operatorname{ad}_{\eta}=L \mathbb{T}_{0}
$$

Remark 3.4 Condition (3.1) holds for an open and dense, full measure set of elements $\eta \in L G$. The assumption that $\overline{\{\exp t \eta: t \in \mathbb{R}\}}$ is a maximal torus holds on a residual full measure set, but fails for a dense set of elements $\eta$ when $\operatorname{rank} G \geq 2$. 
Definition 3.5 The Hopf bifurcation in (2.1) is nonresonant if $\sigma_{0}=\exp \frac{2 \pi}{\omega_{b}} \eta$ is a regular element of $G$. Otherwise, the Hopf bifurcation is resonant.

Example 3.6 If $G$ is abelian (including $G=\mathbf{S O}(2)$ ), we have seen that there are no singular elements, so resonant Hopf bifurcation is impossible according to Definition 3.5.

If $G=\mathbf{S O}(3)$, then by Example $3.2, \sigma_{0}=\exp \frac{2 \pi}{\omega_{b}} \eta$ is singular if and only if it is the identity element. We can write $\eta \in \operatorname{LSO}(3)$ as $\eta=\left(\begin{array}{ccc}0 & -\omega_{r} & 0 \\ \omega_{r} & 0 & 0 \\ 0 & 0 & 0\end{array}\right)$ and so $\sigma_{0}=\left(\begin{array}{ccc}\cos \frac{2 \pi \omega_{r}}{\omega_{b}} & -\sin \frac{2 \pi \omega_{r}}{\omega_{b}} & 0 \\ \sin \frac{2 \pi \omega_{r}}{\omega_{b}} & \cos \frac{2 \pi \omega_{r}}{\omega_{b}} & 0 \\ 0 & 0 & 1\end{array}\right)$. It follows that $\sigma_{0}$ is resonant if and only if $m \omega_{b}=\omega_{r}$ for some $m \geq 1$.

In particular, the resonance condition for $\mathbf{S O}(3)$ is identical in form to Barkley's resonance condition for the Euclidean group $\mathbf{S E}(2)$. In general, the resonance condition depends on the group $G$, see Examples 3.13, 3.14 and 3.15.

Lemma 3.7 (a) Suppose that the Hopf bifurcation is nonresonant. Then there exists $\delta>0$ such that $\sigma_{\lambda}$ lies in a unique maximal torus $\mathbb{T}_{\lambda}$ for $\lambda \in[0, \delta)$, and $\lim _{\lambda \rightarrow 0^{+}} d\left(\mathbb{T}_{\lambda}, \mathbb{T}_{0}\right)=0$.

(b) If the Hopf bifurcation is resonant, then generically there exists $\delta>0$ such that $\sigma_{\lambda}$ lies in a unique maximal torus $\mathbb{T}_{\lambda}$ for $\lambda \in(0, \delta)$. However, generically $\operatorname{dist}\left(\mathbb{T}_{\lambda}, \mathbb{T}_{0}\right) \nrightarrow 0$ and $\operatorname{dist}\left(\mathbb{T}_{0}, \mathbb{T}_{\lambda}\right) \nrightarrow 0$ as $\lambda \rightarrow 0^{+}$.

Proof Part (a) follows from openness of $U$ and Proposition 3.3. Part (b) follows from open-density of $U$ and the comments after Proposition 3.3.

Recall that $H\left(\sigma_{\lambda}\right)=\overline{\left\{\sigma_{\lambda}^{n}: n \in \mathbb{Z}\right\}}$. For $\sigma_{\lambda}$ regular, we have $H\left(\sigma_{\lambda}\right) \subset \mathbb{T}_{\lambda}$. Moreover, generically (for all but countably many parameters in a generic one-parameter family) $H\left(\sigma_{\lambda}\right)=\mathbb{T}_{\lambda}$.

We defined $E$ and $P_{\lambda}$ in (2.3). Note that $E=\mathbb{T}_{0} \times\{0\}$. Also $P_{\lambda}$ is a $H\left(\sigma_{\lambda}\right)$-invariant flow-invariant subset of $G \times X$ with $P_{\lambda} / H\left(\sigma_{\lambda}\right) \cong S_{\lambda}$. Generically, $H\left(\sigma_{\lambda}\right)=\mathbb{T}_{\lambda}$ in which case $P_{\lambda} \cong \mathbb{T}_{\lambda} \times S_{\lambda}$ is quasiperiodic with rank $G+1$ independent frequencies.

Theorem 3.8 (a) If the Hopf bifurcation is nonresonant, then $\lim _{\lambda \rightarrow 0^{+}} \operatorname{dist}\left(P_{\lambda}, E\right) \rightarrow 0$ and typically $\lim _{\lambda \rightarrow 0^{+}} d\left(P_{\lambda}, E\right) \rightarrow 0$.

(b) If the Hopf bifurcation is resonant, then typically $\operatorname{dist}\left(P_{\lambda}, E\right) \nrightarrow 0$ and $\operatorname{dist}\left(E, P_{\lambda}\right) \nrightarrow 0$ as $\lambda \rightarrow 0^{+}$.

Proof Part (b) is immediate from Lemma 3.7(b). 
For $\lambda \geq 0$, let $G_{\lambda}=\left\{g_{\lambda}(t): t \geq 0\right\}$. (Note that $\overline{G_{0}}=\mathbb{T}_{0}$.) To prove the first statement in part (a), we must show that $\operatorname{dist}\left(G_{\lambda}, \mathbb{T}_{0}\right) \rightarrow 0$.

Write $G_{\lambda}=\bigcup_{n>0} G_{\lambda}(n)$ where $G_{\lambda}(n)=\left\{g_{\lambda}(t): 2 n \pi / \omega_{b}(\lambda) \leq t \leq 2(n+\right.$ 1) $\left.\pi / \omega_{b}(\lambda)\right\}$. Then for each fixed $n$, we have $d\left(G_{\lambda}(n), G_{0}(n)\right) \rightarrow 0$. In particular $\operatorname{dist}\left(G_{\lambda}(n), \mathbb{T}_{0}\right) \rightarrow 0$ for each $n$.

Now $G_{\lambda}(n)=\sigma_{\lambda}^{n} G_{\lambda}(0)$. By Lemma $3.7(\mathrm{a}), \operatorname{dist}\left(\mathbb{T}_{\lambda}, \mathbb{T}_{0}\right) \rightarrow 0$ and so $\operatorname{dist}\left(\sigma_{\lambda}^{n}, \mathbb{T}_{0}\right) \rightarrow 0$ uniformly in $n$. Hence $\operatorname{dist}\left(G_{\lambda}(n), \mathbb{T}_{0}\right) \rightarrow 0$ uniformly in $n$ so that $\operatorname{dist}\left(G_{\lambda}, \mathbb{T}_{0}\right) \rightarrow 0$ as required.

Moreover, generically $H\left(\sigma_{0}\right)=\mathbb{T}_{0}$. In this case, we have $d\left(H\left(\sigma_{\lambda}\right), \mathbb{T}_{0}\right) \rightarrow 0$, $d\left(G_{\lambda}, \mathbb{T}_{0}\right) \rightarrow 0$, and $d\left(P_{\lambda}, E\right) \rightarrow 0$, completing the proof of part (a).

Remark 3.9 It is natural to consider setwise convergence in Lemma 3.7 and Theorem 3.8. Note that pointwise convergence, $\lim _{\lambda \rightarrow 0^{+}}\left(g_{\lambda}(t), x_{\lambda}(t)\right)=\left(g_{0}(t), 0\right)$ for each fixed $t$, is automatic. On the other hand, it would be too much to require that $\lim _{\lambda \rightarrow 0^{+}} \sup _{t}\left|g_{\lambda}(t)-g_{0}(t)\right|=0$.

For compact Lie groups, our definition of resonance coincides with the definitions in $[9,20]$ :

Proposition 3.10 Suppose that the relative equilibrium in (2.2) undergoes Hopf bifurcation with frequency $\omega_{b}$. Then resonance occurs if and only if $\operatorname{ad}_{\eta}$ has eigenvalues $\pm i m \omega_{b}$ for some $m \geq 1$.

Proof Let $d=\operatorname{rank} G$. Then $g \in G$ is singular if and only if $\operatorname{dim} \operatorname{ker}\left(\operatorname{Ad}_{g}-I\right)>d$. Taking $g=\exp \frac{2 \pi}{\omega_{b}} \eta$, we have $\operatorname{Ad}_{g}=\operatorname{expad} \frac{2 \pi}{\omega_{b}} \eta$, and so $\operatorname{ad}_{\frac{2 \pi}{\omega_{b}} \eta}$ has at least $d+1$ eigenvalues in $2 \pi i \mathbb{Z}$. It follows from (3.1) that $\operatorname{ad}_{\eta}$ has precisely $d$ zero eigenvalues leaving at least two eigenvalues of the form $\pm i m \omega_{b}, m \geq 1$.

Remark 3.11 For $G$ abelian, all elements are regular and hence resonance never occurs. In contrast, resonant Hopf bifurcation is always of codimension two when the compact Lie group $G$ is not abelian.

To see this, first note that the Hopf bifurcation itself is codimension one. It suffices to show that the set of $\omega_{b}>0$ for which $\exp \frac{2 \pi}{\omega_{b}} \eta$ is singular consists of isolated points but is nonempty. Since $G$ is not abelian, $\operatorname{dim} G>\operatorname{rank} G$, so it follows from (3.1) that $\operatorname{ad}_{\frac{2 \pi}{\omega_{b}} \eta}$ has nonzero eigenvalues. Since $G$ is compact, these eigenvalues are purely imaginary. As $\omega_{b}$ varies, the eigenvalues pass through $2 \pi i \mathbb{Z}-\{0\}$ yielding the required codimension two points by Proposition 3.10.

Definition 3.12 In the case of resonant Hopf bifurcation, let $m \geq 1$ be least such that $\pm i m \omega_{b}$ are eigenvalues of $\operatorname{ad}_{\eta}$. Then the resonance is $m$ 'th order.

Example 3.13 Consider the group $G=\mathbf{S O}(n)$ for $n=2 d$ even. Let $S$ denote the set of matrices that have the block diagonal structure $R_{\theta_{1}} \oplus \cdots \oplus R_{\theta_{d}}$, where $R_{\theta_{j}} \in \mathbf{S O}(2)$ 
has eigenvalues $e^{ \pm i \theta_{j}}, 0 \leq \theta \leq \pi$. Obviously $S \in Z(g)$ for all $g \in S$ and it is easily verified that $Z(g)^{0}=S$ if and only if $\theta_{1}, \ldots, \theta_{d}$ are all distinct. In particular $S$ is a maximal torus and $\operatorname{rank} \mathbf{S O}(n)=d=n / 2$.

We can suppose that $\eta \in L S$ with eigenvalues $\pm i \omega_{j}$ where $\omega_{1}>\omega_{2}>\cdots>\omega_{d}>0$. Then $\exp \frac{2 \pi}{\omega_{b}} \eta$ is singular if and only if $\frac{2 \pi}{\omega_{b}} \omega_{j}= \pm \frac{2 \pi}{\omega_{b}} \omega_{k} \bmod 2 \pi$ for some $j, k$ yielding the condition for resonance

$$
m \omega_{b}= \pm \omega_{j} \pm \omega_{k}, \quad m \geq 1,1 \leq j<k \leq d .
$$

(Of course, in the special case $G=\mathbf{S O}(2)$, the resonance condition is never satisfied.)

Example 3.14 The case $G=\mathbf{S O}(n)=\mathbf{S O}(2 d+1)$ with $n$ odd is similar to the even case, taking $S$ to be the set of matrices $R_{\theta_{1}} \oplus \cdots \oplus R_{\theta_{d}} \oplus 1$. We obtain $\operatorname{rank} \mathbf{S O}(n)=$ $d=(n-1) / 2$ and the condition for regularity is that the $\theta_{j}$ are all distinct and different from 0 .

This time, $\eta$ has eigenvalues $\pm i \omega_{j}$ and 0 where $\omega_{1}>\omega_{2}>\cdots>\omega_{d}>0$. We obtain the conditions for resonance

$$
m \omega_{b}= \pm \omega_{j} \pm \omega_{k}, \quad m \geq 1,1 \leq j<k \leq d
$$

and

$$
m \omega_{b}= \pm \omega_{j}, \quad m \geq 1,1 \leq j \leq d .
$$

Example 3.15 Consider the special unitary group $G=\mathbf{S U}(n), n \geq 2$, consisting of $n \times n$ complex matrices $A$ satisfying $A^{*} A=I$ and $\operatorname{det} A=1$. Let $S$ denote the $(n-1)$-torus consisting of diagonal complex matrices $g \in \mathbf{S U}(n)$. The diagonal entries $\alpha_{j} \in \mathbb{C}, 1 \leq j \leq n$ satisfy $\left|\alpha_{j}\right|=1$ and $\alpha_{1} \cdots \alpha_{n}=1$. Obviously, $S \in Z(g)$ for all $g \in S$ and it is easily verified that $Z(g)^{0}=S$ if and only if the $\alpha_{j}$ are distinct. In particular, $S$ is a maximal torus and $\operatorname{rank} \mathbf{S U}(n)=n-1$.

We can suppose that $\eta \in L S$, so that $\eta$ is diagonal with distinct nonzero entries $i \omega_{j}$ where $\omega_{1}+\cdots+\omega_{n}=0$. Hence $\exp \frac{2 \pi}{\omega_{b}} \eta$ is singular if and only if $m \omega_{b}=\omega_{j}-\omega_{k}$ for some $m \in \mathbb{Z}-\{0\}$ and $1 \leq j<k \leq n$.

In the case $n=2$, the diagonal matrices $g \in S$ have $\alpha_{2}=\bar{\alpha}_{1}$ and so are singular if and only if $g= \pm I$. Hence $U=\mathbf{S U}(2)-\{ \pm I\}$. Moreover $\eta \in L S$ has diagonal entries $\pm \omega_{r}$ and the condition for resonance reduces to $m \omega_{b}=2 \omega_{r}$. In particular, resonances for $\mathbf{S U}(2)$ occur twice as often as for $\mathbf{S O}(3)$ corresponding to the fact that $\mathbf{S U}(2)$ is the double cover of $\mathbf{S O}(3)$.

\section{Solving the skew product equations}

We return to the skew-product equations (2.1). The Hopf bifurcation in the $\dot{x}$ equation yields a branch of periodic solutions $x_{\lambda}(t)$ with period $2 \pi / \omega_{b}(\lambda)$ where $\omega_{b}(\lambda)$ depends smoothly on $\lambda$ and $\omega_{b}(0)=\omega_{b}$. Substituting into the $\dot{g}$ equation, 
we obtain $\dot{g}=g \xi\left(x_{\lambda}(t), \lambda\right)$. If $G$ is abelian, then we can solve explicitly to obtain $g_{\lambda}(t)=\exp \int_{0}^{t} \xi\left(x_{\lambda}(s), \lambda\right) d s$. In the nonabelian case, we generalise the approach of Chan [6] making use of the normal form theory of Fiedler \& Turaev [9]. The reduction to normal form is carried out in Subsection 4.1. The solutions to the normal form equations are presented in the nonresonant and resonant cases in Subsections 4.2 and 4.3 respectively.

\subsection{Reduction to normal form}

Suppressing the parameter $\lambda$ momentarily and following Fiedler \& Turaev [9], we transform the skew product equations (2.1) by a near-identity change of coordinates

$$
x \mapsto P(x), \quad g \mapsto g \exp Q(x),
$$

where $P: \mathbb{R}^{2} \rightarrow \mathbb{R}^{2}$ and $Q: \mathbb{R}^{2} \rightarrow L G$ are polynomial maps. The transformation $P$ puts $\dot{x}=f(x)$ into the standard Birkhoff normal form up to any finite order:

$$
\dot{z}=\tilde{f}\left(|z|^{2}, \lambda\right) z
$$

where $\tilde{f}: \mathbb{R} \times \mathbb{R} \rightarrow \mathbb{C}$ is a polynomial. This has the solution $\dot{z}=r(\lambda) e^{i \omega_{b}(\lambda) t}$ where $r(\lambda)^{2}$ and $\omega_{b}(\lambda)$ are smooth functions of $\lambda$, with $\omega_{b}(\lambda)=\omega_{b}+O(\lambda)$ and $r(\lambda)^{2}=$ $a \lambda+O\left(\lambda^{2}\right)$ where typically $a \neq 0$.

On adding the terms in the tail, it is well-known that $z_{\lambda}(t)$ persists as a periodic solution with frequency $\omega_{b}(\lambda)=\omega_{b}+O(\lambda)$.

Next we turn to the $\dot{g}$-equation. Define $Y_{m}=\operatorname{ker}\left(\operatorname{ad}_{\eta}+i m \omega_{b} I\right) \subset L G$ (complexified).

Lemma 4.1 Through any required order, $\xi$ can be transformed to the following normal forms:

Nonresonant case $\xi(z, \bar{z}, \lambda)=h_{1}\left(|z|^{2}, \lambda\right)$ where $h_{1}: \mathbb{R} \times \mathbb{R} \rightarrow L \mathbb{T}_{0}$.

m'th order resonance $\xi(z, \bar{z}, \lambda)=h_{1}\left(|z|^{2}, \lambda\right)+z^{m} h_{2}\left(|z|^{2}, \lambda\right)+\bar{z}^{m} \bar{h}_{2}\left(|z|^{2}, \lambda\right)$ where $h_{1}: \mathbb{R} \times \mathbb{R} \rightarrow L \mathbb{T}_{0}, h_{2}: \mathbb{R} \times \mathbb{R} \rightarrow Y_{m}$.

Proof Let $k, \ell \geq 0$ and $\zeta \in L G$. Following Fiedler \& Turaev [9, Definition 1.1], we say that the monomial $z^{k} \bar{z}^{\ell} \zeta$ is "resonant" if $\zeta$ is an eigenvector for $-\operatorname{ad}_{\eta}$ with eigenvalue $i \omega_{b}(k-\ell)$. It follows from Fiedler \& Turaev [9, Theorem 1.2] that through arbitrarily high order, all terms in $\xi$ can be removed except for the resonant monomials.

Zero eigenvalues $(k=\ell)$ are characterised in (3.1) and yield the $h_{1}$ terms. In the nonresonant case, there are no further eigenvalues of the form $i \omega_{b}(k-\ell)$. In the case of an $m$ 'th order resonance, we have eigenvalues $\pm i \omega_{b} m$, so taking $k-\ell= \pm m$ yields the $h_{2}$ terms. 


\subsection{Nonresonant case}

In this subsection, we use Lemma 4.1 to solve the nonresonant case.

Theorem 4.2 In the nonresonant case, for each $k \geq 1$,

$$
g_{\lambda}(t)=\exp \left\{t \eta_{k}(\lambda)\right\} \exp \left\{Q_{k}(t, \lambda)+E_{k}(t, \lambda)\right\},
$$

where

(i) $\eta_{k}(\lambda), Q_{k}(t, \lambda), E_{k}(t, \lambda) \in L G$ depend smoothly on $\sqrt{\lambda}$,

(ii) $Q_{k}=O(\sqrt{\lambda})$ and $E_{k}=o\left(\lambda^{k}\right)$,

(iii) $Q_{k}(0, \lambda) \equiv 0$ and $E_{k}(0, \lambda) \equiv 0$,

(iv) $t \rightarrow Q_{k}(t, \lambda)$ is $2 \pi / \omega_{b}(\lambda)$-periodic,

(v) $\eta_{k}(\lambda)=\eta+\sqrt{\lambda} \operatorname{ad}_{b} \eta+O(\lambda) \in L \mathbb{T}_{\lambda}=\operatorname{Ad}_{\exp q(\lambda)} L \mathbb{T}_{0}$, where $q(\lambda)=\sqrt{\lambda} b+O(\lambda)$ and $b$ is a general element of $L G$.

In particular, the spatiotemporal generator $\sigma_{\lambda}=g_{\lambda}\left(2 \pi / \omega_{b}(\lambda)\right)$ satisfies

$$
\sigma_{\lambda}=\exp \left\{\frac{2 \pi}{\omega_{b}(\lambda)} \operatorname{Ad}_{\exp q(\lambda)}[\eta+O(\lambda)]\right\}=\exp \left\{\frac{2 \pi}{\omega_{b}(\lambda)}\left[\eta+\sqrt{\lambda} \operatorname{ad}_{b} \eta+O(\lambda)\right]\right\} .
$$

Proof Working first in the normal form coordinates, and neglecting the tail terms, we substitute the $2 \pi / \omega_{b}(\lambda)$-periodic solution $z_{\lambda}(t)$ into the normal form for $\xi$. In the nonresonant case, we have

$$
\dot{g}=g h_{1}\left(r(\lambda)^{2}, \lambda\right)=g \hat{\eta}(\lambda),
$$

where $\hat{\eta}(\lambda) \in L \mathbb{T}_{0}$ is a smooth function of $\lambda$ and $\hat{\eta}(0)=\eta$. We can solve explicitly to obtain the normal form solution $g_{\lambda}(t)=\exp (t \hat{\eta}(\lambda))$.

Transforming back to the original coordinates, $g_{\lambda}(t)=\exp (t \hat{\eta}(\lambda)) \exp (-q(t, \lambda))$ where $q(t, \lambda) \in L G$ is $2 \pi / \omega_{b}(\lambda)$-periodic with leading term of order $\sqrt{\lambda}$. In order to satisfy the initial condition $g_{\lambda}(0)=e$, we premultiply by $\exp (q(0, \lambda))$ obtaining (by equivariance) the solution

$$
g_{\lambda}(t)=\exp (q(0, \lambda)) \exp (t \hat{\eta}(\lambda)) \exp (-q(0, \lambda)) \exp (Q(t, \lambda)),
$$

where $Q(t, \lambda)$ is $2 \pi / \omega_{b}(\lambda)$-periodic with leading term of order $\sqrt{\lambda}$, and $Q(0, \lambda) \equiv 0$. Hence, $g_{\lambda}(t)=\exp (t \eta(\lambda)) \exp (Q(t, \lambda))$ where $\eta(\lambda)=\operatorname{Ad}_{\exp (q(0, \lambda))} \hat{\eta}(\lambda)$. Writing $q(\lambda)=$ $q(0, \lambda)=\sqrt{\lambda} b+O(\lambda)$, we obtain $\eta(\lambda)=\eta+\sqrt{\lambda} \operatorname{ad}_{b} \eta+O(\lambda)$.

Finally, we note that the Floquet representation for $g_{\lambda}(t)$ may change when the tail terms are included. However, $g_{\lambda}(t)$ itself will be unchanged except up to a (nonperiodic) term $E_{k}=o\left(\lambda^{k}\right)$.

A useful abbreviation is to say that $g_{\lambda}(t)=\exp (t \eta(\lambda)) \exp \{Q(t, \lambda)\}$ beyond all orders where 
(i) $\eta(\lambda), Q(t, \lambda) \in L G$ depend smoothly on $\sqrt{\lambda}$,

(ii) $t \rightarrow Q(t, \lambda)$ is $2 \pi / \omega_{b}(\lambda)$-periodic, $Q=O(\sqrt{\lambda})$, and $Q(0, \lambda) \equiv 0$,

(iii) $\eta(\lambda)=\eta+\sqrt{\lambda} \operatorname{ad}_{b} \eta+O(\lambda) \in L \mathbb{T}_{\lambda}=\operatorname{Ad}_{\exp q(\lambda)} \mathbb{T}_{0}$, where $q(\lambda)=\sqrt{\lambda} b+O(\lambda)$ and $b$ is a general element of $L G$.

\subsection{Resonant case}

In this subsection, we assume that there is an $m$ 'th order resonance. Substitute $z_{\lambda}(t)=r(\lambda) e^{i \omega_{b}(\lambda) t}$ into the normal form for $\xi$ in Lemma 4.1 (neglecting tail terms) to obtain the equation

$$
\dot{g}=g\left[\eta(\lambda)+\lambda^{m / 2} e^{i m \omega_{b}(\lambda) t} \Omega_{0}(\lambda)+\lambda^{m / 2} e^{-i m \omega_{b}(\lambda) t} \bar{\Omega}_{0}(\lambda)\right],
$$

where

$$
\eta(\lambda) \in L \mathbb{T}_{0}, \quad \Omega_{0}(\lambda) \in Y_{m}=\operatorname{ker}\left(\operatorname{ad}_{\eta}+i m \omega_{b} I\right) .
$$

Viewing $\left(\eta(\lambda), \omega_{b}(\lambda)\right) \in L \mathbb{T}_{0} \times \mathbb{R}$ as parameters, generically there is a codimension one set near $\left(\eta(0), \omega_{b}(0)\right)=\left(\eta, \omega_{b}\right)$ for which $\pm i m \omega_{b}(\lambda)$ are eigenvalues of $\operatorname{ad}_{\eta(\lambda)}$. This is the set of resonant parameters.

At such a resonance, working modulo terms of order $\lambda^{m / 2+1}$ in (4.1), we can replace $\Omega_{0}(\lambda)$ by the appropriate eigenfunction (still denoted $\Omega_{0}(\lambda)$ ) of $\operatorname{ad}_{\eta(\lambda)}$ so that $\operatorname{ad}_{\eta(\lambda)} \Omega_{0}(\lambda)=-i m \omega_{b}(\lambda) \Omega_{0}(\lambda)$. Let $\Omega(\lambda)=\Omega_{0}(\lambda)+\bar{\Omega}_{0}(\lambda)$.

Theorem 4.3 Suppose that $\pm i m \omega_{b}(\lambda)$ are eigenvalues of $\operatorname{ad}_{\eta(\lambda)}$ for $\lambda \geq 0$. Then

$$
g_{\lambda}(t)=\exp \left\{t\left[\rho(\lambda)+\lambda^{m / 2} \Omega(\lambda)\right]\right\} \exp \{Q(t, \lambda)+E(t, \lambda)\}
$$

where

(i) $\rho(\lambda), \Omega(\lambda), Q(t, \lambda), E(t, \lambda) \in L G$ depend smoothly on $\sqrt{\lambda}$,

(ii) $Q=O(\sqrt{\lambda})$ and $E=o\left(\lambda^{m / 2}\right)$,

(iii) $Q(0, \lambda) \equiv 0$ and $E(0, \lambda) \equiv 0$,

(iv) $t \rightarrow Q(t, \lambda)$ is $2 \pi / \omega_{b}(\lambda)$-periodic,

(v) $\mathbb{T}_{\lambda}=\overline{\{\exp t \Omega(\lambda), t \in \mathbb{R}\}}$ is a maximal torus for $\lambda \geq 0$,

(vi) $\rho(\lambda) \in L \mathbb{T}_{\lambda} \cap \operatorname{Ad}_{\exp q(\lambda)} L \mathbb{T}_{0}$, where $q(\lambda)=\sqrt{\lambda} b+O(\lambda)$ and $b$ is a general element of $L G$.

In particular, the spatiotemporal generator $\sigma_{\lambda}=g_{\lambda}\left(2 \pi / \omega_{b}(\lambda)\right)$ satisfies

$$
\sigma_{\lambda}=\exp \left\{\frac{2 \pi}{\omega_{b}(\lambda)}\left[\rho(\lambda)+\lambda^{m / 2} \Omega(\lambda)+o\left(\lambda^{m / 2}\right)\right]\right\}
$$


Proof By construction, $\operatorname{Ad}_{\exp t \eta(\lambda)} \Omega_{0}(\lambda)=e^{-i m \omega_{b}(\lambda) t} \Omega_{0}(\lambda)$, and the solution to (4.1) is

$$
g_{\lambda}(t)=\exp \left\{t \lambda^{m / 2} \Omega(\lambda)\right\} \exp \{t \eta(\lambda)\} .
$$

Let $\mathbb{T}_{\lambda}$ denote the maximal torus defined by $\Omega(\lambda)$. Note that $\exp \{t \Omega(\lambda)\} \in$ $Z\left(\exp \frac{2 \pi}{\omega_{b}(\lambda)} \eta(\lambda)\right)$ for all $t$. It follows that $\exp \frac{2 \pi}{\omega_{b}(\lambda)} \eta(\lambda)$ lies in $Z(\exp \{t \Omega(\lambda)\})$ for all $t$, and hence lies in $Z\left(\mathbb{T}_{\lambda}\right)=\mathbb{T}_{\lambda}$. In addition, $\exp \frac{2 \pi}{\omega_{b}(\lambda)} \eta(\lambda) \in \mathbb{T}_{0}$. Hence, we have shown that $\exp \frac{2 \pi}{\omega_{b}(\lambda)} \eta(\lambda) \in \mathbb{T}_{\lambda} \cap \mathbb{T}_{0}$.

Choose $\rho(\lambda) \in L \mathbb{T}_{\lambda} \cap L \mathbb{T}_{0}$ so that $\exp \left\{\frac{2 \pi}{\omega_{b}(\lambda)} \eta(\lambda)\right\}=\exp \left\{\frac{2 \pi}{\omega_{b}(\lambda)} \rho(\lambda)\right\}$. Then $g_{\lambda}(t)$ can be written in the form

$$
g_{\lambda}(t)=\exp \left\{t\left[\rho(\lambda)+\lambda^{m / 2} \Omega(\lambda)\right]\right\} R(t, \lambda),
$$

where $R(t, \lambda)=\exp \{-t \rho(\lambda)\} \exp \{t \eta(\lambda)\}$ is $2 \pi / \omega_{b}(\lambda)$-periodic.

Passing back to the original coordinates and arguing as in the proof of Theorem 4.2 , we obtain a solution of the form

$$
g_{\lambda}(t)=\exp \left\{t\left[\rho(\lambda)+\lambda^{m / 2} \Omega(\lambda)\right]\right\} \exp \{Q(t, \lambda)\}
$$

where $\rho(\lambda) \in \operatorname{Ad}_{\exp q(\lambda)} L \mathbb{T}_{0}$ and $\Omega(\lambda) \in \operatorname{Ad}_{q(\lambda)} L \mathbb{T}_{\lambda}$. Tail terms leave $g_{\lambda}(t)$ unchanged beyond all orders.

Remark 4.4 Note that the family of elements $\rho(\lambda) \in L G$ correspond to singular elements of $G$. When $G=\mathbf{S O}(3)$, the only singular element of $G$ is the identity and so $\rho(\lambda) \equiv 0$. This accounts for the simplified form of the solutions in $[6,7,20]$. The new phenomenon for more general groups is that after the bifurcation some of the $\mathbb{T}_{0}$ frequencies are retained, namely those in common with the new torus $\mathbb{T}_{\lambda}$. The other $\mathbb{T}_{0}$ frequencies are switched off, while the non-common frequencies in $\mathbb{T}_{\lambda}$ set in at the slow $\lambda^{m / 2}$ rate.

Of course, Theorem 4.3 is vacuous for abelian groups such as $\mathbf{S O}(2)$, since there are no resonant Hopf bifurcations.

Acknowledgments DC wishes to think the University of Surrey and EPSRC for financial support via a Doctoral Studentship. The research of IM was supported in part by a Leverhulme Research Fellowship.

\section{References}

[1] D. Barkley. A model for fast computer simulation of waves in excitable media. Physica D 49 (1991) 61-70.

[2] D. Barkley. Euclidean symmetry and the dynamics of rotating spiral waves. Phys. Rev. Lett. 72 (1994) 164-167. 
[3] D. Barkley, M. Kness and S. Tuckerman. Spiral-wave dynamics in a simple model of excitable media: The transition from simple to compound rotation. Phys. Rev. A 42 (1990) 2489-2492.

[4] T. Bröcker and T. tom Dieck. Representations of Compact Lie Groups. Grad. Texts in Math. 98, Springer, New York, 1985.

[5] D. Chan. A normal form approach to nonresonant and resonant Hopf bifurcation from relative equilibria. Ph. D. Thesis, University of Surrey, 2007.

[6] D. Chan. Hopf bifurcations from relative equilibria in spherical geometry. $J$. Differential Equations 226 (2006) 118-134.

[7] A. Comanici. Transition from rotating waves to modulated rotating waves on a sphere. SIAM J. Appl. Dyn. Syst. 5 (2006) 759-782.

[8] B. Fiedler, B. Sandstede, A. Scheel and C. Wulff. Bifurcation from relative equilibria to non-compact group actions: Skew products, meanders, and drifts. Doc. Math. J. DMV 1 (1996) 479-505.

[9] B. Fiedler and D. V. Turaev. Normal forms, resonances, and meandering tip motions near relative equilibria of Euclidean group actions. Arch. Rational Mech. Anal. 145 (1998) 129-159.

[10] M. Golubitsky, V. G. LeBlanc and I. Melbourne. Meandering of the spiral tip an alternative approach. J. Nonlinear Sci. 7 (1997) 557-586.

[11] M. Golubitsky, V. G. LeBlanc and I. Melbourne. Hopf bifurcation from rotating waves and patterns in physical space. J. Nonlinear Sci. 10 (2000) 69-101.

[12] A. Karma. Meandering transition in two-dimensional excitable media. Phys. Rev. Lett. 65 (1990) 2824-2827.

[13] M. Krupa. Bifurcations of relative equilibria. SIAM J. Math. Anal. 21 (1990) $1453-1486$.

[14] G. Li, Q. Ouyang, V. Petrov and H. L. Swinney. Transition from simple rotating chemical spirals to meandering and traveling spirals. Phys. Rev. Lett. 77 (1996) 2105-2108.

[15] A. L. Onishchik and E. B. Vinberg. Lie groups and Lie algebras. III. Encyclopaedia of Mathematical Sciences 42, Springer-Verlag, Berlin, 1991.

[16] B. Sandstede, A. Scheel and C. Wulff. Dynamics of spiral waves on unbounded domains using center-manifold reductions. J. Differential Equations 141 (1997) 122-149. 
[17] B. Simon. Representations of Finite and Compact Groups. Grad. Studies in Math. 10, Amer. Math. Soc., Providence, 1996.

[18] A. T. Winfree. Scroll-shaped waves of chemical activity in three dimensions. Science 181 (1973) 937-939.

[19] C. Wulff. Theory of meandering and drifting spiral waves in reaction-diffusion systems. Dissertation, FU Berlin, 1996.

[20] C. Wulff. Transitions from relative equilibria to relative periodic orbits. Doc. Math. J. DMV 5 (2000) 227-274. 